\title{
RNA-Seq Data Mining: Downregulation of NeuroD6 Serves as a Possible Biomarker for Alzheimer's Disease Brains
}

\author{
Jun-ichi Satoh, Yoji Yamamoto, Naohiro Asahina, Shouta Kitano, and Yoshihiro Kino \\ Department of Bioinformatics and Molecular Neuropathology, Meiji Pharmaceutical University, 2-522-1 Noshio, Kiyose, \\ Tokyo 204-8588, Japan \\ Correspondence should be addressed to Jun-ichi Satoh; satoj@my-pharm.ac.jp
}

Received 24 July 2014; Accepted 17 November 2014; Published 8 December 2014

Academic Editor: Lance A. Liotta

Copyright (C) 2014 Jun-ichi Satoh et al. This is an open access article distributed under the Creative Commons Attribution License, which permits unrestricted use, distribution, and reproduction in any medium, provided the original work is properly cited.

\begin{abstract}
Alzheimer's disease (AD) is the most common cause of dementia worldwide with no curative therapies currently available. Previously, global transcriptome analysis of $\mathrm{AD}$ brains by microarray failed to identify the set of consistently deregulated genes for biomarker development of $\mathrm{AD}$. Therefore, the molecular pathogenesis of $\mathrm{AD}$ remains largely unknown. Whole RNA sequencing (RNA-Seq) is an innovative technology for the comprehensive transcriptome profiling on a genome-wide scale that overcomes several drawbacks of the microarray-based approach. To identify biomarker genes for AD, we analyzed a RNA-Seq dataset composed of the comprehensive transcriptome of autopsized $\mathrm{AD}$ brains derived from two independent cohorts. We identified the core set of 522 genes deregulated in $\mathrm{AD}$ brains shared between both, compared with normal control subjects. They included downregulation of neuronal differentiation 6 (NeuroD6), a basic helix-loop-helix (bHLH) transcription factor involved in neuronal development, differentiation, and survival in AD brains of both cohorts. We verified the results of RNA-Seq by analyzing three microarray datasets of $\mathrm{AD}$ brains different in brain regions, ethnicities, and microarray platforms. Thus, both RNA-Seq and microarray data analysis indicated consistent downregulation of NeuroD6 in AD brains. These results suggested that downregulation of NeuroD6 serves as a possible biomarker for AD brains.
\end{abstract}

\section{Introduction}

Alzheimer's disease (AD) is the most common cause of dementia worldwide affecting the elderly population, characterized by the hallmark pathology of amyloid- $\beta(\mathrm{A} \beta)$ deposition, neurofibrillary tangle (NFT) formation, and extensive neurodegeneration in the brain. The complex interaction between multiple genetic and environmental factors affecting various molecular pathways plays a key role in the pathogenesis of $\mathrm{AD}$ [1]. With regard to environmental factors, disturbed homeostasis of dietary metals, such as copper, aluminum, and iron, confers an increased risk of $\mathrm{AD}[2,3]$. With respect to genetic factors, genome-wide association studies (GWAS), composed of large cohorts of $\mathrm{AD}$ and controls, identified numerous common variants but with smaller risks associated with development of late-onset $\mathrm{AD}$ [4]. They include complement component receptor 1 (CR1), bridging integrator $1(B I N 1)$, clusterin $(C L U)$, phosphatidylinositol binding clathrin assembly protein (PICALM), membrane-spanning 4-domains, subfamily A, member $4 \mathrm{~A} /$ membrane-spanning 4-domains, subfamily A, member 6E (MS4A4/MS4A6E), $\mathrm{CD} 2$-associated protein $(C D 2 A P), \mathrm{CD} 33$ molecule (CD33), EPH receptor A1 (EPHA1), and ATP-binding cassette, subfamily A, member 7 (ABCA7) [4]. More recently, wholeexome sequencing (WES) studies discovered rare functional variants located in the genes encoding $\mathrm{A} \beta$ precursor protein $(A P P)$, triggering receptor expressed on myeloid cells 2 (TREM2), and phospholipase D3 (PLD3), exhibiting a much greater contribution to protection or development of $\mathrm{AD}$ [5-7]. However, at present, the central molecular mechanism underlying neurodegeneration in $\mathrm{AD}$ remains largely unknown. Therefore, no curative therapies based on the molecular pathogenesis of $\mathrm{AD}$ are currently available.

The completion of the Human Genome Project in 2003 allows us to systematically study disease-associated profiles of the whole human genome. Particularly, microarray technologies enable us not only to identify disease-specific molecular signatures and biomarkers for diagnosis and prediction of prognosis but also to characterize druggable targets for effective therapy. Actually, global transcriptome analysis 
of postmortem $\mathrm{AD}$ brains by microarray has identified a battery of genes aberrantly regulated in $\mathrm{AD}$, whose role has not been previously predicted in its pathogenesis [8]. They include reduced expression of kinases/phosphatases, cytoskeletal proteins, synaptic proteins, and neurotransmitter receptors in NFT-bearing CA1 neurons [9], downregulation of neurotrophic factors and upregulation of proapoptotic molecules in the hippocampal CA1 region [10], disturbed sphingolipid metabolism in various brain regions during progression of $\mathrm{AD}$ [11], and overexpression of the AMPA receptor GluR2 subunit in synaptosomes of the prefrontal cortex [12]. However, previous studies failed to identify the set of definite biomarker genes, whose expression is consistently deregulated in $\mathrm{AD}$ brains across different studies [8]. The failure in reproducibility of the results is attributable to differences in study designs and samples, including the quality of RNA, disease stages, brain regions, cellular diversities, ethnicities, and microarray platforms [13].

Recently, the revolution of the next-generation sequencing (NGS) technology has made a great impact on the field of genome research. Whole RNA sequencing (RNA-Seq) serves as an innovative tool for the comprehensive transcriptome profiling on a genome scale in a high-throughput and quantitative manner $[14,15]$. RNA-Seq clarifies the unbiased expression of the complete set of transcripts at a single base resolution, including splice junctions and fusion genes, by providing digital gene expression levels with high reproducibility. RNA-Seq enables us to characterize the complex transcriptome, composed of mRNAs, noncoding RNAs, and small RNAs, theoretically at a single cell level, by aligning sequencing reads on reference genomes or assembling them de novo without references. For these reasons, RNA-Seq overcomes several drawbacks intrinsic to the microarraybased approach that is hampered by the difficulty in detection of novel transcripts and splice variants, the poor sensitivity of rare transcripts, and high backgrounds due to cross hybridization.

To identify biomarker genes relevant to the molecular pathogenesis of $\mathrm{AD}$, we analyzed publicly available RNA-Seq datasets, composed of the comprehensive transcriptome of autopsied AD brains derived from two independent cohorts. First, we identified the core set of 522 genes deregulated in $\mathrm{AD}$ brains overlapping between both. Then, we verified the results of RNA-Seq by analyzing three independent microarray datasets of AD brains that are different in brain regions, ethnicities, and microarray platforms. Consequently, we found consistent downregulation of neuronal differentiation 6 (NeuroD6), a bHLH transcription factor involved in neuronal development and differentiation, serving as a possible biomarker for $\mathrm{AD}$ brains.

\section{Materials and Methods}

2.1. RNA-Seq Datasets of AD Brains. To identify a comprehensive set of differentially expressed genes (DEGs) in the brains of $\mathrm{AD}$ patients compared with normal control (NC) subjects, we investigated FASTQ-formatted files of RNA-Seq datasets retrieved from the DDBJ Sequence Read Archive
(DRA) (https://trace.ddbj.nig.ac.jp/DRASearch) under the accession number of SRA060572. It consisted of 15 separate samples derived from two independent cohorts, studied by the researchers in Emory University, Atlanta, here abbreviated as EMU, and by those in the University of Kentucky, Lexington, abbreviated as UKY [16]. The EMU dataset contains transcriptome of the frontal cortex isolated from three male and two female $\mathrm{AD}$ patients with age $=71.0 \pm 8.2$ years and postmortem interval (PMI) $=13.8 \pm 7.2$ hours and two male and two female NC subjects with age $=60.8 \pm 3.3$ years and PMI $=9.0 \pm 2.6$ hours. The UKY dataset contains transcriptome of the frontal cortex isolated from three female $\mathrm{AD}$ patients with age $=81.7 \pm 3.2$ years and $\mathrm{PMI}=2.2 \pm$ 0.4 hours and three female NC subjects with age $=85.0 \pm$ 1.0 years and PMI $=2.8 \pm 0.6$ hours. The information on the Braak stage of AD pathology [17] is not available for any cases. In these experiments, total RNA was purified by oligo (dT) beads and converted to cDNA for PCR amplification using SMARTer PCR cDNA Synthesis Kit (Clontech). Then, PCR products were fragmented and processed for DNA library preparation via PCR amplification using NEBNext DNA Library Prep Master Mix Set for Illumina (New England BioLabs). The final DNA library products with size $\sim 200 \mathrm{bp}$ were prepared for paired-end sequencing on HiSeq 2000 (Illumina).

After removing poly-A tails and low quality reads from the original data, we mapped short read data on the human genome reference sequence hg19 by using TopHat2.0.9 (http://ccb.jhu.edu/software/tophat/index.shtml). The expression levels were transformed into fragments per kilobase of exon per million mapped fragments (FPKM). We identified DEGs that satisfy the significance expressed as $q$-value representing FDR-adjusted $P$ value $<0.05$ by using Cufflinks2.1.1 (http://cufflinks.cbcb.umd.edu).

2.2. Microarray Datasets of $A D$ Brains. To verify the results of RNA-Seq data analysis, we investigated three distinct microarray datasets of $\mathrm{AD}$ brains retrieved from Gene Expression Omnibus (GEO) (http://www.ncbi.nlm.nih.gov/ geo/) under accession numbers of GSE1297, GSE5281, and GSE11829. The GSE1297 dataset contains transcriptome of postmortem hippocampal CA1 tissues studied on a Human Genome U133A Array containing 22,215 transcripts (Affymetrix), and the data were normalized by the Microarray Analysis Suite 5.0 (MAS5) algorithm [18]. The samples were collected by the researchers in UKY. They were prepared from 31 age-matched individuals, composed of nine NC subjects (age $=85.3 \pm 8.0$ years; male $=7$, female $=2$ ), seven patients with incipient $\mathrm{AD}$ (age $=91.9 \pm 6.2$ years; male $=2$, female $=5$ ), eight with moderate $\mathrm{AD}$ (age $=83.4 \pm 3.2$ years; male $=2$, female $=6$ ), and seven with severe $\mathrm{AD}$ (age $=84.0$ \pm 10.6 years; male $=2$, female $=5$ ). The clinical stage of $\mathrm{AD}$ was defined by the Mini-Mental State Examination (MMSE) score as follows: the control (the score $>25$ ), incipient (20$26)$, moderate (14-19), and severe (<14) AD. The information on the Braak stage of AD pathology is not available for any cases. 
The GSE5281 dataset, alternatively named steph-affyhuman 433773, contains transcriptome of laser microdissection (LCM)-captured layer III neurons derived from various brain regions studied on a Human Genome U133 Plus 2.0 Array containing 47,400 transcripts (Affymetrix), and the data were normalized by MAS5 [19]. The samples were collected by the researchers in AD Centers of Arizona, Duke University, and Washington University. We studied the gene expression profile of cortical neurons in the superior frontal gyrus, which were isolated from 11 age-matched NC subjects $($ age $=79.3 \pm 10.2$ years; male $=7$, female $=4)$ and $23 \mathrm{AD}$ patients $($ age $=79.2 \pm 7.5$ years; male $=13$, female $=10)$. The information on the Braak stage of AD pathology is not available for any cases.

The GSE36980 dataset contains transcriptome of postmortem brain tissues isolated from frontal and temporal cortices and the hippocampus studied on a Human Gene 1.0 ST Array containing 28,869 genes (Affymetrix), and the data were normalized by the robust multiarray average (RMA) algorithm [20]. The samples were collected by the researchers in Kyushu University, Japan, for the Hisayama study. We studied the gene expression profile of the hippocampus, derived from ten non-AD controls (age $=77.0 \pm 9.0$ years; male $=5$, female $=5$ ) and seven AD patients (age $=92.9 \pm$ 6.1 years; male $=3$, female $=4$ ). The information on the Braak stage of $\mathrm{AD}$ pathology is not available for any cases.

To evaluate the statistically significant difference in gene expression levels between $\mathrm{AD}$ and $\mathrm{NC}$ or non-AD groups, we performed a two-tailed Welch $t$-test by using TTEST function of Excel. In some experiments, we performed receiver operating characteristic (ROC) analysis by using SPSS version 19 (IBM).

2.3. Molecular Network Analysis. We imported Entrez Gene IDs of DEGs into the Functional Annotation tool of Database for Annotation, Visualization and Integrated Discovery (DAVID) v6.7 [21]. DAVID extracts gene ontology (GO) terms enriched in the set of imported genes and identifies relevant pathways constructed by Kyoto Encyclopedia of Genes and Genomes (KEGG). The results are followed by statistical evaluation with the modified Fisher exact test corrected by multiple comparison tests. We considered $P$ value $<0.05$ after Bonferroni's correction as significant. KEGG is a publicly accessible knowledgebase that contains 337,524 manually curated pathways that cover a wide range of metabolic, genetic, environmental, and cellular processes and human diseases [22].

We also imported Entrez Gene IDs of DEGs into the Core Analysis tool of Ingenuity Pathways Analysis (IPA) (Ingenuity Systems). IPA is a commercial knowledgebase that contains approximately 3,000,000 biological and chemical interactions with definite scientific evidence. By uploading the list of Gene IDs, the network-generation algorithm identifies focused genes integrated in global molecular pathways and networks. IPA calculates the score $P$ value that reflects the statistical significance of association between the genes and the pathways and networks by Fisher's exact test. We considered $P$ value $<0.05$ by Fisher's exact test as significant.

\section{Results}

3.1. RNA-Seq Data Analysis of AD Brains. By RNA-Seq data analysis with the combination of TopHat and Cufflinks, we studied transcriptome of the frontal cortex of $\mathrm{AD}$ and $\mathrm{NC}$ derived from two distinct cohorts named EMU and UKY. We identified 587,301 and 766,998 consensus transcripts in total from datasets of EMU and UKY, respectively. Among them, we identified 1,226 DEGs for EMU and 2,625 DEGs for UKY that satisfy $q$-value (FDR-corrected $P$ value) $<0.05$ and fold change greater than 2.0 or smaller than 0.5 , when compared between $\mathrm{AD}$ and $\mathrm{NC}$ groups. Then, we extracted the core set of 522 DEGs overlapping between both cohorts, composed of 470 downregulated and 52 upregulated genes in $\mathrm{AD}$ (see Supplementary Table 1 of the Supplementary Material available online at http://dx.doi.org/10.1155/2014/123165). Thus, downregulated genes greatly outnumbered upregulated ones in AD brains. Top 20 genes are listed in Table 1. Notably, the expression of neuronal differentiation 6 (NeuroD6), a brainspecific basic helix-loop-helix (bHLH) transcription factor [23], is greatly reduced at fold changes 0.095 for EMU and 0.159 for UKY in AD brains ( $q=0.0023$ for EMU and 0.0006 for UKY) (Table 1, italicized). Furthermore, lipid phosphate phosphatase-related protein type 4 (LPPR4; PRG1), a direct target gene of NeuroD6 [24], was also downregulated in $\mathrm{AD}$ brains of both cohorts (Supplementary Table 1). We identified totally 60 differentially spliced genes in the frontal cortex of $\mathrm{AD}$, when the data derived from both cohorts were combined, although none of them were shared between both (Supplementary Table 2).

DAVID revealed that the set of 470 DEGs downregulated in the frontal cortex of $\mathrm{AD}$ are relevant to $\mathrm{GO}$ terms of "synaptic transmission" (GO:0007268; $P=2.545 E-17$ corrected by Bonferroni) and "transmission of nerve impulse" (GO:0019226; $P=1.778 E-15$ corrected by Bonferroni). They are also relevant to the KEGG pathway named "neuroactive ligand-receptor interaction" (hsa04080; $P=0.0004$ corrected by Bonferroni) (Figure 1). In contrast, the set of 52 genes upregulated in $\mathrm{AD}$ were not significantly associated with any GO terms or KEGG pathways. IPA showed that the core set of 522 DEGs have a significant relationship with top two different functional networks defined as "Cell-ToCell Signaling and Interaction, Nervous System Development and Function, Neurological Disease" $(P=1.00 E-71)$ and "Hereditary Disorder, Neurological Disease, Psychological Disorders" $(P=1.00 E-71)$ (Figure 2). Taken together, these results suggest that a battery of the genes essential for neuronal interactions is coordinately downregulated in $\mathrm{AD}$ brains.

3.2. Microarray Data Analysis of AD Brains. To verify the results of RNA-Seq data analysis, we studied three distinct microarray datasets of AD brains numbered GSE1297, GSE5281, and GSE36980. We compared the core set of 522 DEGs of RNA-Seq with DEGs extracted from microarray datasets. First, we studied the GSE5281 dataset composed of transcriptome of LCM-captured cortical neurons in the superior frontal gyrus. We identified the set of $215 \mathrm{DEGs}$ 


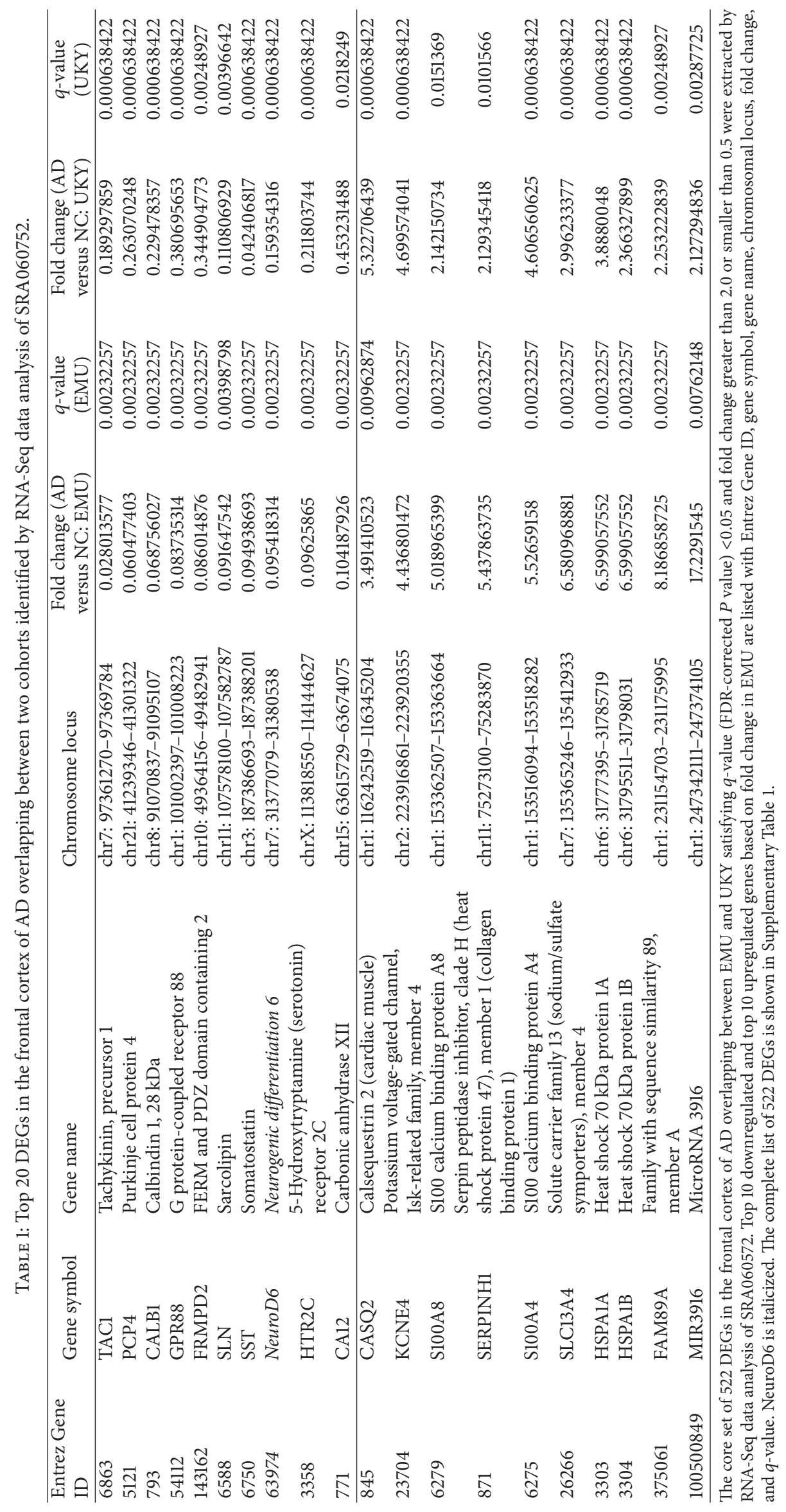


TABLE 2: The set of 15 genes DEGs downregulated in cortical neurons of the superior frontal gyrus of AD identified by microarray data analysis of GSE5281 corresponding to RNA-Seq data analysis of SRA060752.

\begin{tabular}{|c|c|c|c|c|}
\hline $\begin{array}{l}\text { Entrez Gene } \\
\text { ID }\end{array}$ & Gene symbol & Gene name & $\begin{array}{c}\text { Fold change } \\
\text { (AD versus NC) }\end{array}$ & $P$ value \\
\hline 116 & ADCYAP1 & Adenylate cyclase activating polypeptide 1 (pituitary) & 0.069636923 & $1.26039 E-07$ \\
\hline 6750 & SST & Somatostatin & 0.078547047 & $2.49899 E-05$ \\
\hline 10777 & ARPP21 & cAMP-regulated phosphoprotein, $21 \mathrm{kDa}$ & 0.1474134 & $4.52823 E-06$ \\
\hline 6511 & SLC1A6 & $\begin{array}{l}\text { Solute carrier family } 1 \text { (high affinity } \\
\text { aspartate/glutamate transporter), member } 6\end{array}$ & 0.156759363 & $4.74541 E-05$ \\
\hline 728192 & LINC00460 & Long intergenic non-protein-coding RNA 460 & 0.171656016 & $8.49002 E-07$ \\
\hline 891 & CCNB1 & Cyclin B1 & 0.182817517 & $1.34014 E-05$ \\
\hline 523 & ATP6V1A & $\begin{array}{l}\text { ATPase, } \mathrm{H}+\text { transporting, lysosomal } 70 \mathrm{kDa}, \mathrm{V} 1 \\
\text { subunit A }\end{array}$ & 0.190442377 & $8.45909 E-05$ \\
\hline 7991 & TUSC3 & Tumor suppressor candidate 3 & 0.200399391 & $3.74354 E-05$ \\
\hline 1741 & DLG3 & Discs, large homolog 3 (Drosophila) & 0.222930685 & $8.59311 E-06$ \\
\hline 63974 & NeuroD6 & Neurogenic differentiation 6 & 0.237572737 & $6.60728 E-05$ \\
\hline 3382 & ICA1 & Islet cell autoantigen $1,69 \mathrm{kDa}$ & 0.249762845 & $2.41638 E-05$ \\
\hline 844 & CASQ1 & Calsequestrin 1 (fast-twitch, skeletal muscle) & 0.271852315 & $3.03481 E-05$ \\
\hline 9577 & BRE & $\begin{array}{l}\text { Brain and reproductive organ-expressed (TNFRSF1A } \\
\text { modulator) }\end{array}$ & 0.306075086 & $9.92354 E-05$ \\
\hline 84900 & RNFT2 & Ring finger protein, transmembrane 2 & 0.306171729 & $4.52424 E-05$ \\
\hline 9515 & STXBP5L & Syntaxin binding protein 5 -like & 0.360338998 & $8.58457 E-05$ \\
\hline
\end{tabular}

The set of 215 DEGs in LCM-captured frontal cortex neurons of AD satisfying $P$ value $<0.0001$ by two-tailed $t$-test and fold change greater than 2 or smaller than 0.5 were extracted by microarray data analysis of GSE5281. Among them, the set of 15 genes corresponding to the core set of 522 DEGs identified by RNASeq data analysis of SRA060572 are listed with Entrez Gene ID, gene symbol, gene name, fold change, and $P$ value. NeuroD6 is italicized. The complete set of 215 DEGs are shown in Supplementary Table 3.

compared between $\mathrm{AD}$ and $\mathrm{NC}$ groups, including 210 downregulated and 5 upregulated genes in $\mathrm{AD}$ (Supplementary Table 3). Because downregulated genes greatly outnumbered upregulated classes, we thereafter focused on the downregulated set. Among them, we found that 15 genes correspond to the core set of 522 DEGs of RNA-Seq (Table 2). Notably, the expression of NeuroD6 was reduced at fold change $=0.238$ in purified cortical neurons of the superior frontal gyrus in AD brains $(P=0.000066$, Table 2 , italicized). In contrast, the levels of expression of NeuroD1 were not significantly different between $\mathrm{AD}$ and NC brains $(P=0.530$, not shown). ROC analysis indicated that the area under the ROC curve (AUC) is 0.893 for NeuroD6 and 0.474 for NeuroD1, and the levels of sensitivity and specificity for discrimination between $\mathrm{AD}$ and NC are acceptable for NeuroD6 $(P=2.494 E-04)$ but unacceptable for NeuroD1 $(P=0.811)$ (Supplementary Figure 1).

Next, we attempted to answer the question whether downregulation of NeuroD6 serves as a possible biomarker for diagnosis of $\mathrm{AD}$ by brain transcriptome profiling, regardless of differences in brain regions, microarray platforms, or ethnicities of samples. We analyzed two more datasets of transcriptome of postmortem hippocampal tissues isolated from Caucasian (GSE1297 on Human Genome U133A Array) or Japanese (GSE36980 on Human Gene 1.0 ST Array) AD patients. From the GSE1297 dataset, we identified the set of 131 DEGs downregulated in the hippocampal CA1 region at fold change of severe AD versus NC $<0.6$ (Supplementary Table 4). We found that 25 genes of $131 \mathrm{DEGs}$ correspond to the core set of $522 \mathrm{DEG}$ of RNA-Seq (Table 3). They also included NeuroD6 whose expression levels are reduced in Caucasian $\mathrm{AD}$ brains during progression of $\mathrm{AD}$ at fold change $=0.569$ for the comparison between severe $\mathrm{AD}$ and NC $(P=0.0072$, Table 3 , italicized $)$. From the GSE11829 dataset, we identified the set of 31 DEGs downregulated in the region-unrestricted hippocampus of Japanese AD patients, compared with non-AD controls (Supplementary Table 5). We found that 12 genes of 31 DEGs correspond to the core set of 522 DEGs of RNA-Seq (Table 4). Again, they included NeuroD6 whose expression levels are reduced in AD brains at fold change $=0.433$ for the comparison between $\mathrm{AD}$ and non-AD ( $P=0.0016$, Table 4 , italicized). Taken together, these observations suggest that downregulation of NeuroD6 serves as a fairly universal biomarker for diagnosis of $\mathrm{AD}$ by brain transcriptome profiling, regardless of differences in brain regions, microarray platforms, or ethnicities of samples.

\section{Discussion}

Previously, a number of microarray-based transcriptome studies of $\mathrm{AD}$ brains failed to identify the set of consistently deregulated genes across different studies [8]. RNASeq serves as an innovative technology for the comprehensive transcriptome profiling on a genome scale in a highthroughput and quantitative manner $[14,15]$. To identify biomarker genes relevant to the molecular pathogenesis of $\mathrm{AD}$, we first studied publicly available RNA-Seq datasets 
TABLE 3: The set of 25 DEGs downregulated in the hippocampal CA1 region during progression of AD identified by microarray data analysis of GSE1297 corresponding to RNA-Seq data analysis of SRA060752.

\begin{tabular}{|c|c|c|c|c|}
\hline $\begin{array}{l}\text { Entrez Gene } \\
\text { ID }\end{array}$ & Gene symbol & Gene name & $\begin{array}{l}\text { Fold change } \\
\text { (severe } \mathrm{AD} \text { versus } \mathrm{NC} \text { ) }\end{array}$ & $P$ value \\
\hline 57172 & CAMK1G & Calcium/calmodulin-dependent protein kinase IG & 0.119791216 & 0.00015044 \\
\hline 7447 & VSNL1 & Visinin-like 1 & 0.176659556 & 0.003811085 \\
\hline 10368 & CACNG3 & Calcium channel, voltage-dependent, gamma subunit 3 & 0.212906942 & 0.000645324 \\
\hline 55711 & FAR2 & Fatty acyl CoA reductase 2 & 0.230090829 & 0.00066547 \\
\hline 10769 & PLK2 & Polo-like kinase 2 & 0.264329482 & 0.001588852 \\
\hline 9331 & B4GALT6 & $\begin{array}{l}\text { UDP-Gal: betaGlcNAc beta 1,4-galactosyltransferase, } \\
\text { polypeptide } 6\end{array}$ & 0.284246871 & 0.005450676 \\
\hline 55312 & RFK & Riboflavin kinase & 0.326351561 & 0.000250327 \\
\hline 5274 & SERPINI1 & $\begin{array}{l}\text { Serpin peptidase inhibitor, clade I (neuroserpin), member } \\
1\end{array}$ & 0.337526915 & 0.006332563 \\
\hline 9079 & LDB2 & LIM domain binding 2 & 0.340999225 & 0.003326867 \\
\hline 1268 & CNR1 & Cannabinoid receptor 1 (brain) & 0.344653589 & 0.008057005 \\
\hline 5579 & PRKCB & Protein kinase $\mathrm{C}$, beta & 0.345004889 & 0.002186833 \\
\hline 63982 & ANO3 & Anoctamin 3 & 0.372084936 & 0.008600399 \\
\hline 81831 & NETO2 & Neuropilin (NRP) and tolloid- (TLL-) like 2 & 0.393977566 & 0.000965658 \\
\hline 440270 & GOLGA8B & Golgin A8 family, member B & 0.422538873 & 0.004711934 \\
\hline 23236 & PLCB1 & Phospholipase C, beta 1 (phosphoinositide-specific) & 0.43589904 & 0.001873881 \\
\hline 27324 & TOX3 & TOX high mobility group box family member 3 & 0.450425735 & 0.002724595 \\
\hline 6000 & RGS7 & Regulator of G-protein signaling 7 & 0.451971533 & 0.006045425 \\
\hline 138046 & RALYL & RALY RNA binding protein-like & 0.453463038 & 0.001029166 \\
\hline 5530 & РPР3CA & Protein phosphatase 3 , catalytic subunit, alpha isozyme & 0.461727097 & 0.001385837 \\
\hline 1020 & CDK5 & Cyclin-dependent kinase 5 & 0.464742579 & 0.00340998 \\
\hline 3751 & KCND2 & $\begin{array}{l}\text { Potassium voltage-gated channel, Shal-related subfamily, } \\
\text { member } 2\end{array}$ & 0.489787298 & 0.004105453 \\
\hline 29114 & TAGLN3 & Transgelin 3 & 0.536481218 & 0.00215522 \\
\hline 7534 & YWHAZ & $\begin{array}{l}\text { Tyrosine 3-monooxygenase/tryptophan } \\
\text { 5-monooxygenase activation protein, zeta polypeptide }\end{array}$ & 0.556167619 & 0.006101886 \\
\hline 63974 & NeuroD6 & Neurogenic differentiation 6 & 0.568549476 & 0.007199422 \\
\hline 2744 & GLS & Glutaminase & 0.591660135 & 0.007897416 \\
\hline
\end{tabular}

The set of 131 DEGs downregulated in the hippocampal CA1 region among incipient, moderate, and severe AD and NC groups by one-way ANOVA satisfying $P$ value $<0.01$ and fold change of severe AD versus NC smaller than 0.6 were extracted by microarray data analysis of GSE1297. Among them, the set of 25 genes corresponding to the core set of 522 DEGs identified by RNA-Seq data analysis of SRA060572 are listed with Entrez Gene ID, gene symbol, gene name, fold change, and $P$ value. NeuroD6 is italicized. The complete list of 131 DEGs are shown in Supplementary Table 4.

TABLE 4: The set of 12 DEGs downregulated in the hippocampus of Japanese AD patients identified by microarray data analysis of GSE36980 corresponding to RNA-Seq data analysis of SRA060752.

\begin{tabular}{|c|c|c|c|c|}
\hline $\begin{array}{l}\text { Entrez Gene } \\
\text { ID }\end{array}$ & Gene symbol & Gene name & $\begin{array}{c}\text { Fold change } \\
\text { (AD versus non- } A D)\end{array}$ & $P$ value \\
\hline 63974 & NeuroD6 & Neurogenic differentiation 6 & 0.433171474 & 0.001616741 \\
\hline 10368 & CACNG3 & Calcium channel, voltage-dependent, gamma subunit 3 & 0.496685808 & 0.004499281 \\
\hline 5176 & SERPINF1 & $\begin{array}{l}\text { Serpin peptidase inhibitor, clade } \mathrm{F} \text { (alpha- } 2 \\
\text { antiplasmin, pigment epithelium derived factor), } \\
\text { member } 1\end{array}$ & 0.522226034 & 0.00019849 \\
\hline 348980 & $\mathrm{HCN} 1$ & $\begin{array}{l}\text { Hyperpolarization activated cyclic nucleotide-gated } \\
\text { potassium channel } 1\end{array}$ & 0.523810594 & 0.004508668 \\
\hline 5774 & PTPN3 & Protein tyrosine phosphatase, nonreceptor type 3 & 0.54910964 & 0.001537752 \\
\hline 8507 & ENC1 & Ectodermal-neural cortex (with BTB-like domain) & 0.559652363 & 0.003288053 \\
\hline 266722 & HS6ST3 & Heparan sulfate 6-O-sulfotransferase 3 & 0.560440342 & 0.001753288 \\
\hline 2903 & GRIN2A & $\begin{array}{l}\text { Glutamate receptor, ionotropic, N-methyl D-aspartate } \\
2 \mathrm{~A}\end{array}$ & 0.581203611 & 0.002390123 \\
\hline 51299 & NRN1 & Neuritin 1 & 0.590013553 & 0.001828335 \\
\hline 125113 & KRT222 & Keratin 222 pseudogene & 0.592296133 & 0.004941811 \\
\hline 1428 & CRYM & Crystallin, mu & 0.594516424 & 0.002824762 \\
\hline 221692 & PHACTR1 & Phosphatase and actin regulator 1 & 0.597310446 & 0.002464967 \\
\hline
\end{tabular}

The set of 31 DEGs downregulated in the hippocampus of Japanese AD patients satisfying $P$ value $<0.005$ by two-tailed $t$-test and fold change smaller than 0.6 were extracted by microarray data analysis of GSE36980. Among them, the set of 12 genes corresponding to the core set of 522 DEGs identified by RNA-Seq data analysis of SRA060572 are listed with Entrez Gene ID, gene symbol, gene name, fold change, and $P$ value. NeuroD6 is italicized. The complete list of 31 DEGs are shown in Supplementary Table 5. 

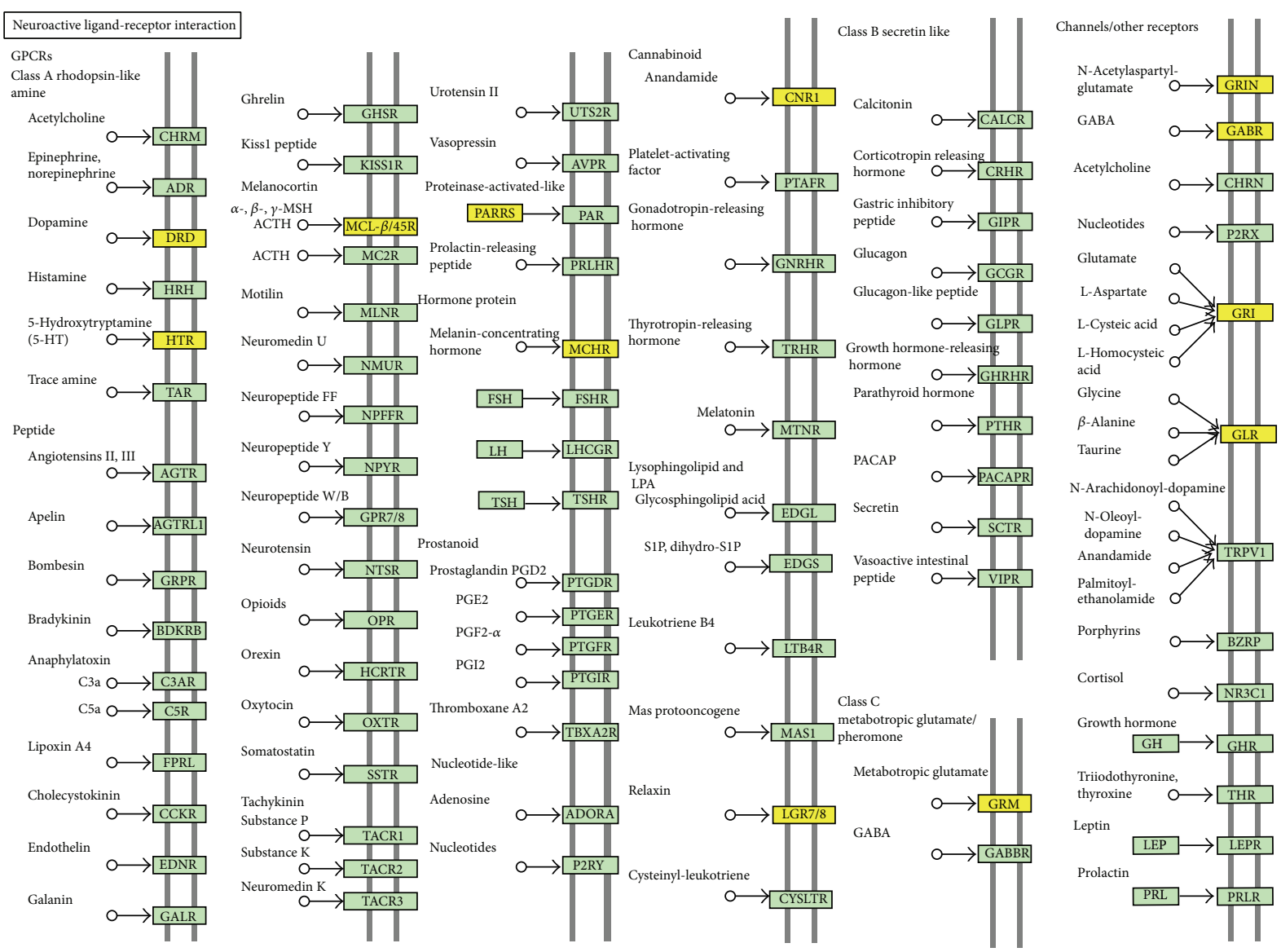

FIGURE 1: KEGG pathway of 470 DEGs downregulated in the frontal cortex of AD identified by RNA-Seq data analysis. Entrez Gene IDs of 470 DEGs downregulated in the frontal cortex of AD identified by RNA-Seq data analysis of SRA060572 were imported into the Functional Annotation tool of DAVID. It extracted the most significant KEGG pathway termed "neuroactive ligand-receptor interaction" (hsa04080) relevant to the set of imported genes. Downregulated genes are colored yellow.

of $\mathrm{AD}$ brain transcriptome derived from two independent cohorts named EMU and UKY. We identified the core set of 522 DEGs consistently deregulated in AD brains of both cohorts. They include 470 downregulated and 52 upregulated genes in $\mathrm{AD}$ brains, relevant to synaptic transmission, neuroactive ligand-receptor interaction, nervous system development, and pathological processes of neuropsychiatric diseases by GO and pathway analysis. Then, we compared the results of RNA-Seq data analysis with those of three distinct microarray datasets of AD brains, which are different in brain regions, ethnicities, and microarray platforms. As a result, we identified consistent downregulation of NeuroD6 in AD brains throughout the datasets studied.

The NeuroD family of bHLH transcription factors, composed of three major members, such as NeuroD1 (BETA2), NeuroD2 (NDRF), and NeuroD6 (NEX1, MATH2, and ATOH2), acts as a differentiation factor for neural precursor cells in the developing central nervous system (CNS) [23]. Each member exhibits an overlapping but distinct spatiotemporal expression profile with partially redundant function in the formation of subpopulations of neurons. A previous study by in situ hybridization showed that NeuroD6 is expressed abundantly in mature adult neurons of the cerebral cortex, the hippocampus, and the cerebellum [25]. Although NeuroD6deficient mice exhibit no obvious defect in development, NeuroD1/NeuroD6 double knockout mice show arrest of terminal differentiation of granule cells in the hippocampus [26]. NeuroD6-expressing progenitor cells located in the subventricular zone have a capacity to differentiate into pyramidal glutamatergic neurons in upper cortical layers [27]. Both NeuroD2 and NeuroD6 regulate axonal fasciculation and proper formation of callosal fiber tracts [28]. NeuroD6 plays a key role in cell fate decision of subtypes of amacrine cells in the retina [29]. Constitutive expression of NeuroD6 triggers neuronal differentiation of PC12 cells, originated from a pheochromocytoma of the rat adrenal medulla, without requirement of nerve growth factor (NGF) [30]. NeuroD6 plays a decisive role in the switch from proapoptotic to antiapoptotic pathways during neuronal differentiation of PC12 cells [31]. Furthermore, NeuroD6 confers tolerance to oxidative stress by inducing antioxidant responses and by increasing the mitochondrial biomass [32]. Importantly, NeuroD6, by forming a coexpression network module with TBR1, FEZF2, FOXG1, SATB2, and EMX1, plays a key role in development of the human neocortex and hippocampus projection neurons that are severely degenerated in AD brains 


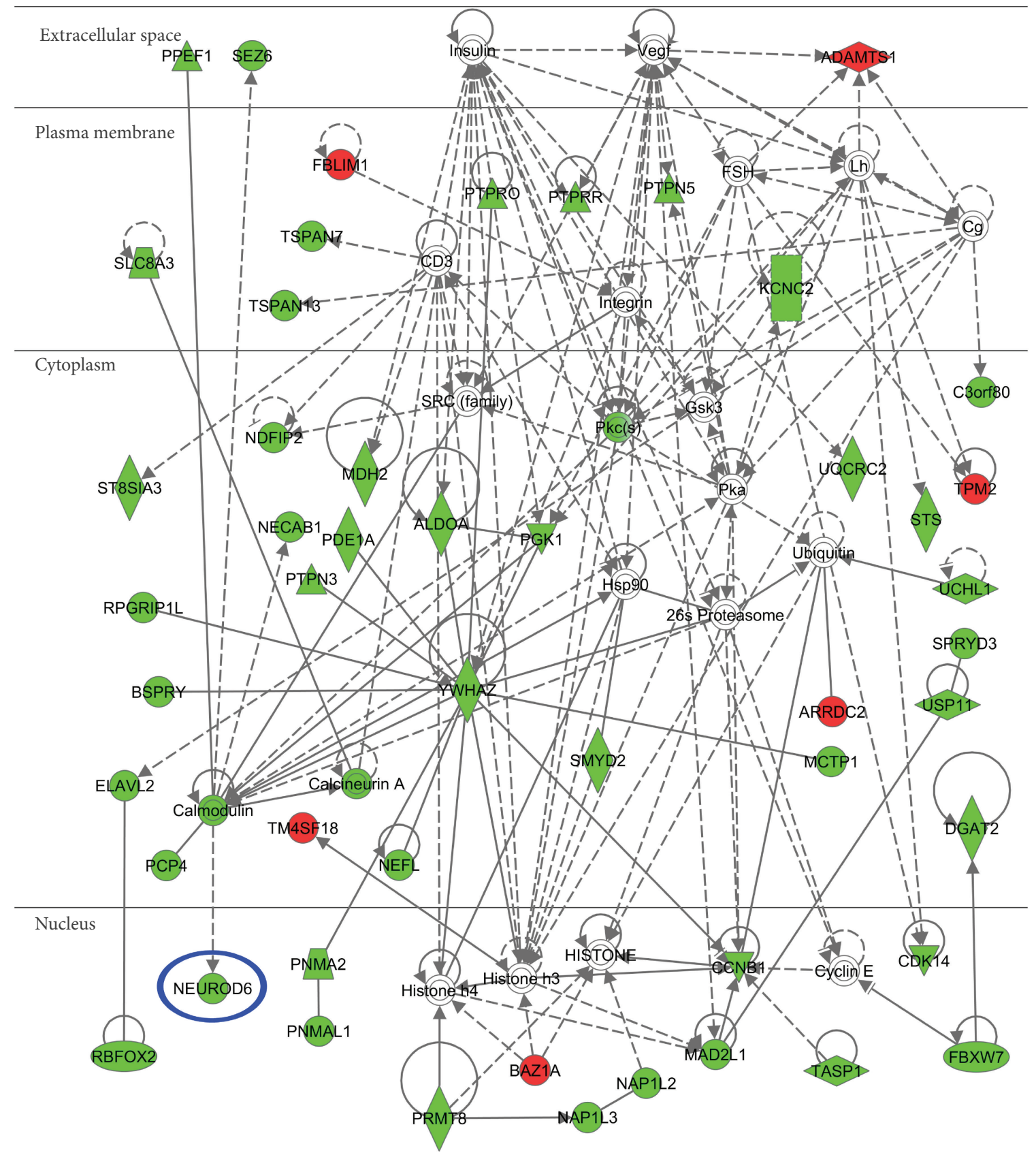

FIGURE 2: IPA pathways of the core set of 522 DEGs in the frontal cortex of AD identified by RNA-Seq data analysis. Entrez Gene IDs of 522 DEGs in the frontal cortex of AD identified by RNA-Seq data analysis of SRA060572 were imported into the Core Analysis tool of IPA. It extracted the most significant functional network termed "Hereditary Disorder, Neurological Disease, Psychological Disorders" relevant to the set of imported genes. Downregulated DEGs are colored green, while upregulated DEGs are colored red. NeuroD6 is highlighted by a blue circle.

[33]. All of these observations suggest that NeuroD6 acts as a key regulator of neuronal development, differentiation, and survival.

Previous studies identified LPPR4 and growth associated protein 43 (GAP43) as direct target genes for NeuroD6 by binding assay to E-boxes located in target gene promoters $[24,34]$. Importantly, we found that the core set of 522 DEGs of RNA-Seq include LPPR as one of the downregulated genes in $\mathrm{AD}$ brains of both cohorts, and the study also identified GAP43 as a downregulated gene in AD brains of the UKY cohort (not shown). Although the precise biological role of NeuroD6 and its target genes in adult human brains remains unknown, the present observations suggest that downregulation of NeuroD6 might be detrimental for neuronal survival 
under stressful conditions caused by extensive accumulation of extracellular $\mathrm{A} \beta$ and intracellular NFT in AD.

In conclusion, the present study using bioinformatics data mining approach suggested that downregulation of NeuroD6 serves as a possible biomarker for diagnosis of $\mathrm{AD}$ by brain transcriptome profiling. Since the sample sizes we studied are apparently small, these findings warrant further validation in larger cohorts of $\mathrm{AD}$ patients and adequate controls performed in a blinded manner.

\section{Conflict of Interests}

The authors declare that there is no conflict of interests regarding the publication of this paper.

\section{Acknowledgments}

The present study was supported by the JSPS KAKENHI (C22500322 and C25430054), the Dementia Drug Development Research Center (DRC) Project, the Ministry of Education, Culture, Sports, Science and Technology (MEXT), Japan, and the grant from the National Center for Geriatrics and Gerontology (NCGC26-20).

\section{References}

[1] M. Gatz, C. A. Reynolds, L. Fratiglioni et al., "Role of genes and environments for explaining Alzheimer disease," Archives of General Psychiatry, vol. 63, no. 2, pp. 168-174, 2006.

[2] J. R. Walton, "Aluminum's involvement in the progression of alzheimer's disease," Journal of Alzheimer's Disease, vol. 35, no. 1, pp. 7-43, 2013.

[3] R. Squitti, M. Siotto, and R. Polimanti, "Low-copper diet as a preventive strategy for Alzheimer's disease," Neurobiology of Aging, vol. 35, no. 2, pp. S40-S50, 2014.

[4] S. L. Rosenthal and M. I. Kamboth, "Late-onset Alzheimer's disease genes and the potentially implicated pathways," Current Genetic Medicine Reports, vol. 2, pp. 85-101, 2014.

[5] T. Jonsson, J. K. Atwal, S. Steinberg et al., "A mutation in APP protects against Alzheimer's disease and age-related cognitive decline," Nature, vol. 487, no. 7409, pp. 96-99, 2012.

[6] R. Guerreiro, A. Wojtas, J. Bras et al., "TREM2 variants in Alzheimer's disease," The New England Journal of Medicine, vol. 368, no. 2, pp. 117-127, 2013.

[7] C. Cruchaga, C. M. Karch, S. C. Jin et al., "Rare coding variants in the phospholipase D3 gene confer risk for Alzheimer's disease," Nature, vol. 505, no. 7484, pp. 550-554, 2014.

[8] J. Cooper-Knock, J. Kirby, L. Ferraiuolo, P. R. Heath, M. Rattray, and P. J. Shaw, "Gene expression profiling in human neurodegenerative disease," Nature Reviews Neurology, vol. 8, no. 9, pp. 518-530, 2012.

[9] S. D. Ginsberg, S. E. Hemby, V. M. Lee, J. H. Eberwine, and J. Q. Trojanowski, "Expression profile of transcripts in Alzheimer's disease tangle-bearing CA1 neurons," Annals of Neurology, vol. 48, no. 1, pp. 77-87, 2000.

[10] V. Colangelo, J. Schurr, M. J. Ball, R. P. Pelaez, N. G. Bazan, and W. J. Lukiw, "Gene expression profiling of 12633 genes in Alzheimer hippocampal CA1: transcription and neurotrophic factor down-regulation and up-regulation of apoptotic and proinflammatory signaling," Journal of Neuroscience Research, vol. 70, no. 3, pp. 462-473, 2002.

[11] P. Katsel, C. Li, and V. Haroutunian, "Gene expression alterations in the sphingolipid metabolism pathways during progression of dementia and Alzheimer's disease: a shift toward ceramide accumulation at the earliest recognizable stages of Alzheimer's disease?" Neurochemical Research, vol. 32, no. 4-5, pp. 845-856, 2007.

[12] C. Williams, R. M. Shai, Y. Wu et al., “Transcriptome analysis of synaptoneurosomes identifies neuroplasticity genes overexpressed in incipient Alzheimer's disease," PLoS ONE, vol. 4, no. 3, Article ID e4936, 2009.

[13] J. J. Chen, H.-M. Hsueh, R. R. Delongchamp, C.-J. Lin, and C.-A. Tsai, "Reproducibility of microarray data: a further analysis of microarray quality control (MAQC) data," BMC Bioinformatics, vol. 8, article 412, 2007.

[14] Z. Wang, M. Gerstein, and M. Snyder, "RNA-Seq: a revolutionary tool for transcriptomics," Nature Reviews Genetics, vol. 10, no. 1, pp. 57-63, 2009.

[15] G. T. Sutherland, M. Janitz, and J. J. Kril, "Understanding the pathogenesis of Alzheimer's disease: will RNA-Seq realize the promise of transcriptomics?" Journal of Neurochemistry, vol. 116, no. 6, pp. 937-946, 2011.

[16] B. Bai, C. M. Hales, P.-C. Chen et al., "U1 small nuclear ribonucleoprotein complex and RNA splicing alterations in Alzheimer's disease," Proceedings of the National Academy of Sciences of the United States of America, vol. 110, no. 41, pp. 16562-16567, 2013.

[17] H. Braak, I. Alafuzoff, T. Arzberger, H. Kretzschmar, and K. del Tredici, "Staging of Alzheimer disease-associated neurofibrillary pathology using paraffin sections and immunocytochemistry," Acta Neuropathologica, vol. 112, no. 4, pp. 389-404, 2006.

[18] E. M. Blalock, J. W. Geddes, K. C. Chen, N. M. Porter, W. R. Markesbery, and P. W. Landfield, “Incipient Alzheimer's disease: microarray correlation analyses reveal major transcriptional and tumor suppressor responses," Proceedings of the National Academy of Sciences of the United States of America, vol. 101, no. 7, pp. 2173-2178, 2004.

[19] W. S. Liang, E. M. Reiman, J. Valla et al., "Alzheimer's disease is associated with reduced expression of energy metabolism genes in posterior cingulate neurons," Proceedings of the National Academy of Sciences of the United States of America, vol. 105, no. 11, pp. 4441-4446, 2008.

[20] M. Hokama, S. Oka, J. Leon et al., "Altered expression of diabetes-related genes in Alzheimer's disease brains: the Hisayama study," Cerebral Cortex, vol. 24, no. 9, pp. 2476-2488, 2014.

[21] D. W. Huang, B. T. Sherman, and R. A. Lempicki, "Systematic and integrative analysis of large gene lists using DAVID bioinformatics resources," Nature Protocols, vol. 4, no. 1, pp. 44-57, 2009.

[22] M. Kanehisa, S. Goto, Y. Sato, M. Kawashima, M. Furumichi, and M. Tanabe, "Data, information, knowledge and principle: back to metabolism in KEGG," Nucleic Acids Research, vol. 42, no. 1, pp. D199-D205, 2014.

[23] M. H. Schwab, S. Druffel-Augustin, P. Gass et al., "Neuronal basic helix-loop-helix proteins (NEX, neuroD, NDRF): spatiotemporal expression and targeted disruption of the NEX gene in transgenic mice," The Journal of Neuroscience, vol. 18, no. 4, pp. 1408-1418, 1998. 
[24] M. Yamada, Y. Shida, K. Takahashi et al., "Prg1 is regulated by the basic helix-loop-helix transcription factor Math2," Journal of Neurochemistry, vol. 106, no. 6, pp. 2375-2384, 2008.

[25] A. Bartholomä and K.-A. Nave, "NEX-1: a novel brain-specific helix-loop-helix protein with autoregulation and sustained expression in mature cortical neurons," Mechanisms of Development, vol. 48, no. 3, pp. 217-228, 1994.

[26] M. H. Schwab, A. Bartholomae, B. Heimrich et al., "Neuronal basic helix-loop-helix proteins (NEX and BETA2/Neuro D) regulate terminal granule cell differentiation in the hippocampus," Journal of Neuroscience, vol. 20, no. 10, pp. 3714-3724, 2000.

[27] S.-X. Wu, S. Goebbels, K. Nakamura et al., "Pyramidal neurons of upper cortical layers generated by NEX-positive progenitor cells in the subventricular zone," Proceedings of the National Academy of Sciences of the United States of America, vol. 102, no. 47, pp. 17172-17177, 2005.

[28] I. Bormuth, K. Yan, T. Yonemasu et al., "Neuronal basic helixloop-helix proteins neurod2/6 regulate cortical commissure formation before midline interactions," The Journal of Neuroscience, vol. 33, no. 2, pp. 641-651, 2013.

[29] J. N. Kay, P. E. Voinescu, M. W. Chu, and J. R. Sanes, "Neurod6 expression defines new retinal amacrine cell subtypes and regulates their fate," Nature Neuroscience, vol. 14, no. 8, pp. 965972, 2011.

[30] M. Uittenbogaard and A. Chiaramello, "Constitutive overexpression of the basic helix-loop-helix Nexl/MATH-2 transcription factor promotes neuronal differentiation of PC12 cells and neurite regeneration," Journal of Neuroscience Research, vol. 67, no. 2, pp. 235-245, 2002.

[31] M. Uittenbogaard and A. Chiaramello, "The basic helix-loophelix transcription factor Nex-1/Math-2 promotes neuronal survival of PC12 cells by modulating the dynamic expression of anti-apoptotic and cell cycle regulators," Journal of Neurochemistry, vol. 92, no. 3, pp. 585-596, 2005.

[32] M. Uittenbogaard, K. K. Baxter, and A. Chiaramello, "The neurogenic basic helix-loop-helix transcription factor NeuroD6 confers tolerance to oxidative stress by triggering an antioxidant response and sustaining the mitochondrial biomass," $A S N$ Neuro, vol. 2, no. 2, Article ID e00034, 2010.

[33] H. J. Kang, Y. I. Kawasawa, F. Cheng et al., "Spatio-temporal transcriptome of the human brain," Nature, vol. 478, no. 7370, pp. 483-489, 2011.

[34] M. Uittenbogaard, D. L. Martinka, and A. Chiaramello, "The basic helix-loop-helix differentiation factor Nex1/MATH-2 functions as a key activator of the GAP-43 gene," Journal of Neurochemistry, vol. 84, no. 4, pp. 678-688, 2003. 


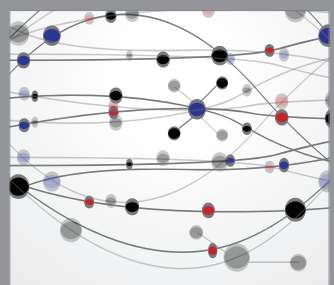

The Scientific World Journal
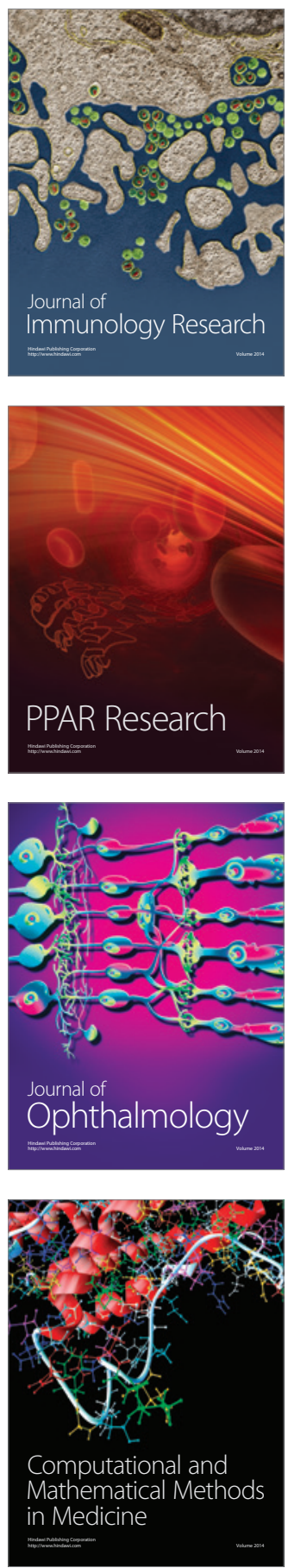

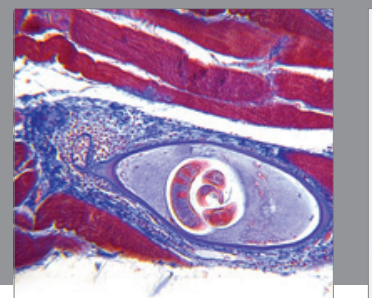

Gastroenterology

Research and Practice
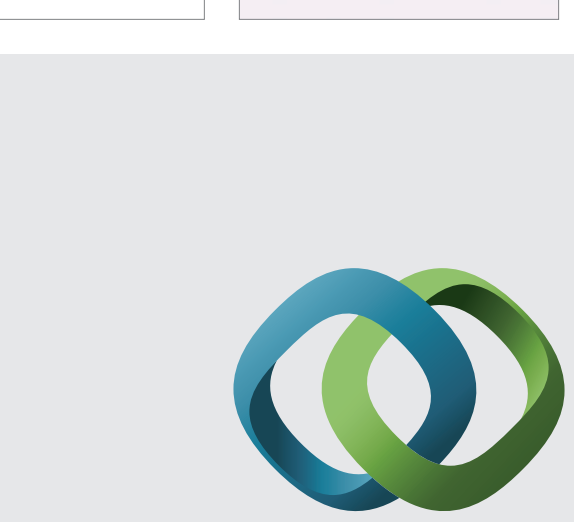

\section{Hindawi}

Submit your manuscripts at

http://www.hindawi.com
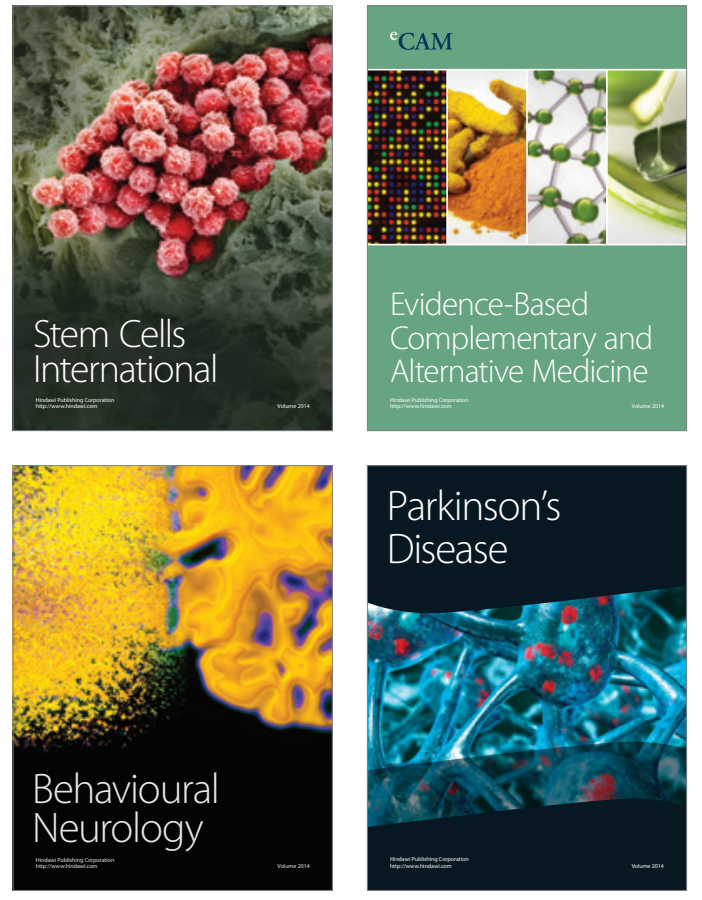
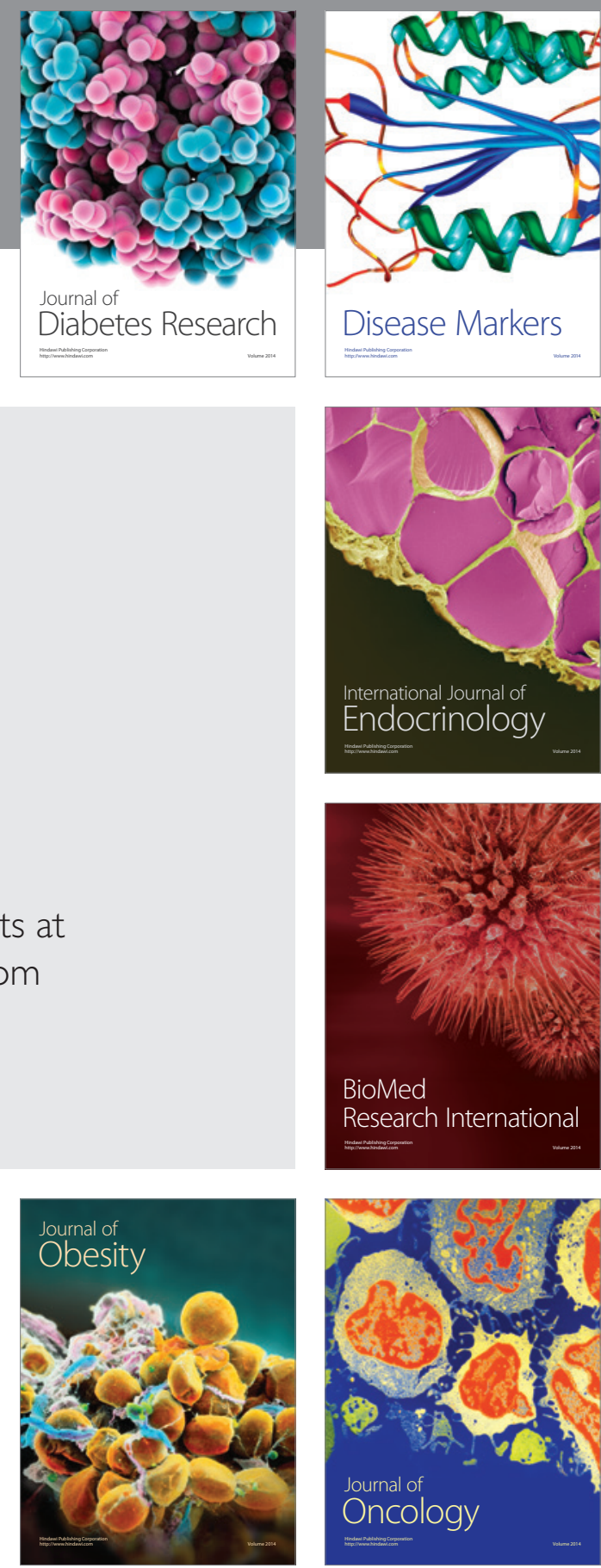

Disease Markers
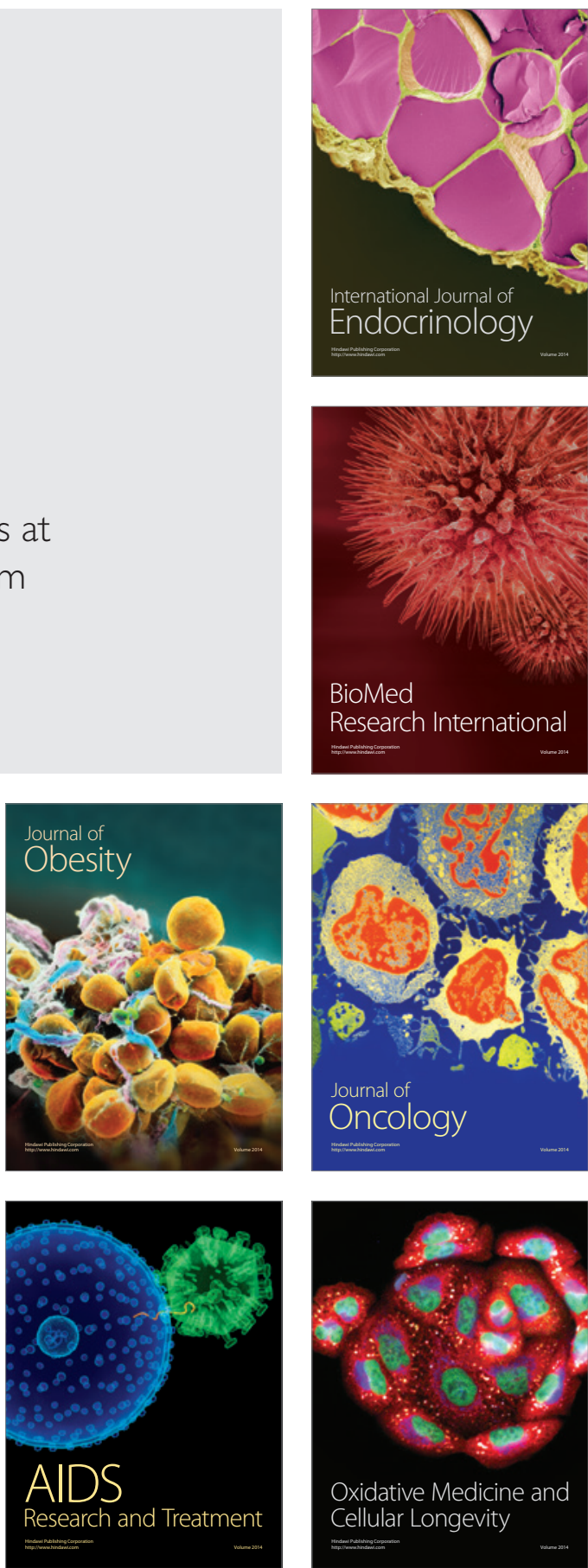